\title{
Is there evidence for automatic processing of spatial and color attributes present in pictures and words?
}

\author{
DENISE CORTIS PARK and DAVID A. MASON \\ University of North Carolina, Charlotte, North Carolina 28223
}

\begin{abstract}
The current experiment studies evidence for automatic processing of color and spatial dimensions present in matched pictures and words. Subjects studied four lists of either line drawings or matched words that varied in color (red or green) and position (left or right side), under one of four encoding conditions. Subjects were instructed to encode (1) only the item, (2) the item and its color, (3) the item and its position, or (4) the item and both color and position. All subjects participated in an unexpected final recognition task in which item recognition and recall for both attributes, regardless of original encoding instructions, were examined. Color memory appeared to be effortful for both pictures and words, as it was at chance level unless subjects were specifically instructed to encode the information. Position was most poorly recalled when subjects attended only to item information, but memory for this dimension was well above chance in all encoding conditions. The position of the line drawings was better recalled than the position of the words. The implications of these results for Hasher and Zacks' (1979) model of automatic processes is discussed.
\end{abstract}

The possibility that certain components of stimuli are remembered automatically is an intriguing one. Although others had noted that some stimulus characteristics were readily remembered, Hasher and Zacks (1979) formally proposed that certain stimulus attributes are automatically encoded and thus remembered without intention. They specified five criteria characteristic of automatic memory processes. The criteria require that (1) a stimulus dimension that is automatically processed is unaffected by age, (2) intention to learn the dimension does not enhance its recall, (3) practice at encoding the dimension does not improve memory, (4) processing the dimension uses minimal capacity, and (5) automatic processes are not reduced when the organism is stressed. Hasher and Zacks suggested that spatial, temporal, and frequency information associated with stimuli are dimensions characterized by automatic encoding processes. They detailed a number of studies that support their conclusions, and since then, other studies have been generated that specifically test Hasher and Zacks' assumptions. However, there are few studies in the literature that systematically investigate evidence for automatic encoding of the same dimension in different types of stimuli. The present study is specifi-

The authors thank the Psychology Department office staff for assistance in typing and preparing this manuscript. Also, we thank Thomas Puglisi for helpful comments. A presentation of this work occurred at the 1980 meeting of the American Psychological Association. Requests for reprints should be sent to D. C. Park, Psychology Department, University of North Carolina at Charlotte, Charlotte, North Carolina 28223. cally concerned with differences in memory for the spatial location of matched pictures and words, as there are conflicting findings about spatial automaticity with respect to stimuli ranging from real-world objects to typed words.

Studies exist that have examined the aging, intentionality, and capacity criteria for automatic processing of spatial information. There is little support for the notion that spatial memory does not change with age, as a number of authors have reported age-related changes (Mandler, Seegmiller, \& Day, 1977; Perlmutter, Metzger, Nezworski, \& Miller, 1981; Von Wright, Gebhard, \& Kartunnen, 1975). However, there is evidence that intentionality to learn spatial material does not facilitate retention of the material, thus confirming a second criterion for automatic processing. Such a finding was reported by Mandler et al. and Von Wright et al. for location of objects and pictures, respectively. Schulman (1973) found intention to learn the location of words actually depressed recall relative to an incidental learning group. However, Acredolo, Pick, and Olsen (1975) found that forewarning children to remember location did improve location recall, so the effects of intentionality to learn on position recall are not consistent. Finally, results have indicated that learning location requires minimal capacity, thus supporting another criterion for automaticity. Both Mandler et al. and Von Wright et al. noted that if subjects attempted to learn spatial location of objects and pictures, memory for the items themselves was unaffected.

In the present study, the criteria of intentionality and capacity are studied with respect to spatial memory 
for words and pictures. Since the Hasher and Zacks (1979) model does not logically require that only the stimulus attributes they specified be automatically processed, the current study also investigates the intentionality and capacity criteria with respect to memory for color. Data indicate that memory for the color of words is effortful. Light and Berger (1974) found that instructing subjects to remember the case (upper or lower) and color of a word improved recall of the attributes, but at the expense of item memory, a finding suggesting capacity and intentionality were required to process the attributes. Light, Berger, and Bardales (1975) reported a similar finding. In a third study, Light and Berger (1976) found evidence for independent as well as effortful memory for color and case attributes of words, so that the finding that it is effortful to remember color is reliable. However, little or no data exist with respect to color memory for pictures. Given the popularity of the notion (as well as data that sometimes support it) that pictures are represented differently in memory from words (Paivio, 1971), the possibility that color memory for pictures might be different from that for words seems plausible.

Thus the current study uses the methodology of Light and Berger (1976) to assess evidence for the automatic processing of the position and color attributes of pictures and words. Subjects are presented with either pictures or words for study and are instructed to study (1) only the item itself, (2) the item and its color, (3) the item and its position, or (4) the item and both attributes. If memory for position and color is automatic, instructions should not affect either item memory or attribute memory. The design also permits us to assess differences in automaticity for each attribute separately, as well as different encoding and/or storage processes that exist for pictures and words.

\section{METHOD}

\section{Subjects}

The subjects were 128 psychology students at the University of North Carolina at Charlotte. They received extra points toward their psychology grades for their voluntary participation.

\section{Stimulus Materials}

The pictorial stimuli used in the study were constructed first. The experimenters selected 134 pictures of everyday objects from the Peabody Picture Vocabulary Test and then conducted an experiment to determine what verbal labels were appropriate for these items. Black-and-white slide transparencies of these pictures were prepared and then presented to a group of 13 subjects. The subjects were instructed to give a single-word name of each object they saw. The experimental design required a total of 112 different pictures, so the 22 items that received the most variable labels were discarded. Of the 112 pictures used, there were 84 to which all 13 subjects gave an identical label. In 19 cases, one subject gave a different but conceptually similar label. And finally, nine pictures had two subjects give dissenting labels (these items were all used as distractors). The overall proportion of agreement collapsed across items and subjects was $97.5 \%$, so the pictures were virtually unambiguous. The labels for the pictures provided by these 13 subjects were then used to construct matching word stimuli. None of these subjects participated in the actual memory experiment.

The experimental design required subjects to study four lists of 16 items, so 64 items were selected for acquisition from the pool of usable pictures. The pictures were then ranked according to the frequency of their labels in Thorndike and Lorge (1944). The items were assigned to the four lists so as to have equivalent numbers of $\mathrm{AA}$ and $\mathrm{A}$ words, and the mean frequency of non-AA and non-A words from Lists 14 were $19.22,18.44,18.11$, and 18.55 , respectively.

Half of the pictures in each list were randomly assigned to be colored red, and half were assigned to be green. Additionally, half were randomly placed in a left position and half on the right, with each color-position combination occurring four times in each list. Also, a second set of acquisition stimulus items was constructed, in which new colors and positions were assigned to the pictures to control for any position or color effects specific to an item. The pictures were then pasted on the left side or right side of $5 \times 8$ in. index cards, and a black-and-white negative slide transparency of the card was developed. Color (red or green) was added to the negative through a diazo process, so the net effect was to produce a slide that had a white-on-red image or a white-on-green image, with the picture located in the left or right field. The word slides were identical in color and location to the pictures, but the picture labels were used instead. Transfer lettering kits $(3 / 8$-in. Helvetica Medium lowercase letters) were used to print the relevant word on to the $5 \mathrm{x}$ $8 \mathrm{in.}$ cards. The words were not typed, because the experimenters wanted the area of each word to approximate the area of each picture and typewritten characters would have been much too small.

All recognition stimuli consisted of a white-on-black image of a picture or word placed in the center of the slide transparency. Recognition items were made for all 64 acquisition items in both picture and word formats, and the remaining items from the initial pool of 112 were used as recognition distractors.

\section{Procedures}

Subjects were tested in groups of one to five, with all subjects in a single group assigned to the same condition. Eight betweengroups conditions were created by crossing the two formats (picture or word) with four types of instructions. Subjects were instructed to study (1) only the item (I condition), (2) the item and its color (I-C condition), (3) the item and its position (I-P condition), or (4) the item, its color, and its position (I-C-P condition). The number of subjects in each of these eight conditions ranged from 12 to 17 . During the instruction period, subjects in each condition saw two sample acquisition items that varied in color and location. Each of the four acquisition lists consisted of 16 items presented individually for $5 \mathrm{sec}$ each. Approximately half of the subjects in each condition received the alternate set of acquisition stimuli that varied in color and position, and list order was rotated within each condition.

Following the presentation of each list, subjects performed a filler task for $60 \mathrm{sec}$ that consisted of crossing out all of the vowels on a sheet of paper with rows of letters printed on it. After the letter-crossing task, subjects participated in an immediate memory test. They saw 12 items $(8$ presented items with 4 new distractors interspersed) presented for 10 sec each. As mentioned earlier, the items were centered white-on-black images. Subjects in the I condition indicated only if they saw the item in the immediately preceding list. I-P, I-C, and I-C-P subjects were also tested for the recall of the particular attribute(s) they were instructed to remember, if they recognized the item. The purpose of immediate recognition was primarily to ensure that subjects learned that the only information they were ever tested for was what they were instructed to remember, so that attention to other dimensions would not occur covertly.

Since only 8 of the 16 presented items were tested immediately, half the subjects in each condition received alternate sets 
of items during immediate recognition, so that across subjects, all items were tested equally often.

Following the fourth presentation of the acquisition/lettercrossing/immediate recognition task, subjects performed a 5 -min filler task before proceeding to final recognition. Subjects received a sheet of paper with the words "Charlotte, North Carolina" typed on it and were instructed to form words out of the letters in the phrase and write them on the sheet.

After this, there was an unexpected final memory test on which subjects saw 96 items: 64 acquisition items interspersed with 32 new distractors. Presentation order was random, and the rate was $10 \mathrm{sec}$. All subjects, regardless of their original encoding instructions, indicated whether or not they recognized each item as one they studied during acquisition by circling "yes" or "no." If they responded affirmatively, they were to indicate the color and position of that item on their response sheet. If they were unsure, they were instructed to make their best guess. Debriefing followed this procedure.

\section{RESULTS AND DISCUSSION}

\section{Immediate Recognition}

Two analyses were conducted on the immediate recognition data: an analysis of the number of hits and an analysis of the number of false alarms. There were no effects significant at or beyond the .01 level in either of these analyses, largely due to subject performance close to ceiling. However, the analysis of variance of hits, which included format (picture or word), instructions, and list as factors, yielded an effect of format significant at the .03 level $[\mathrm{F}(1,107)=4.90, \mathrm{MSe}=2.78]$. This was due to better recognition of pictures than words, with means of 7.32 and 6.97 , respectively (a score of 8.0 was the highest attainable). Also, a small instructions effect was observed $[\mathrm{F}(3,107)=2.72$, $\mathrm{p}<.05, \mathrm{MSe}=2.78]$. As can be seen in Table 1, subjects' performance in the I-only condition was somewhat lower than in the other conditions. One would have expected the reverse, and no explanation is offered for this puzzling finding. There were no significant effects in the analysis of variance of false alarms, due primarily to an overall low rate, as seen in Table 1 (well below a mean rate of one item per subject per condition). Light and Berger (1976) reported a similar lack of effects in their immediate recognition analysis, so these findings were expected.

The last two columns of Table 1 present the probability that subjects correctly recalled the attributes

Table 1

Mean Immediate Recognition Performance on Hits, False Alarms, and Proportion of Attributes Correctly Recalled as a Function of Format (Picture or Word) and Instructions

\begin{tabular}{|c|c|c|c|c|c|c|}
\hline \multirow{3}{*}{$\begin{array}{c}\text { Instruc- } \\
\text { tions }\end{array}$} & \multicolumn{4}{|c|}{ Number of } & \multirow{2}{*}{\multicolumn{2}{|c|}{$\begin{array}{c}\text { Proportion } \\
\text { Attributes } \\
\text { Recalled } \\
\end{array}$}} \\
\hline & \multicolumn{2}{|c|}{ Hits } & \multicolumn{2}{|c|}{$\begin{array}{c}\text { False } \\
\text { Alarms }\end{array}$} & & \\
\hline & Picture & Word & Picture & Word & Picture & Word \\
\hline I Only & 6.77 & 6.87 & .10 & .20 & & \\
\hline $\mathrm{I}-\mathrm{C}$ & 7.64 & 7.25 & .09 & .22 & .83 & .85 \\
\hline I-P & 7.29 & 6.97 & .14 & .17 & .90 & .84 \\
\hline I-C-P & 7.57 & 6.80 & .20 & .32 & .69 & .67 \\
\hline
\end{tabular}

that they were instructed to learn, conditionalized upon an initially correct response. The results were well above chance, as chance performance in the I-P and I-C conditions would be .50 and that in the I-C-P condition would be .25 . A comparison of the I-P and I-C conditions indicated no differences between recall of position and recall of color.

The immediate recognition data offer little insight into the issues with which this study is concerned. However, the analyses indicate a high level of accurate responding, suggesting that subjects were actively attending to the presented information, as instructed.

\section{Final Recognition}

There were five analyses of variance conducted on the final recognition data. Three analyses assessed item recognition $\left(d^{\prime}\right.$, hits, and false alarm analyses of variance), and the other two examined attribute recall for the position and color attributes separately.

To conduct the items analyses, the proportions of hits made as a function of acquisition list and testing during immediate recognition were calculated for each subject. The proportion of false alarms for each subject was also obtained, and finally, the hit and false alarm data were used to calculate a $\mathrm{d}^{\prime}$ value for tested and untested items for each subject. The means for these dependent measures appear in Table 2 as a function of format and instructions. All reported effects were significant at or beyond the .01 level.

The $\mathrm{d}^{\prime}$ analysis of variance included format and instructions as between-groups factors and whether or not an item was tested initially in immediate recognition as a within-subjects measure. The analysis yielded a significant format effect $[\mathrm{F}(1,107)=27.03, \mathrm{MSe}=.96]$, due to a traditional pictorial superiority effect (mean $\mathrm{d}^{3}$ values of 3.35 and 2.67 for pictures and words, respectively). The only other significant effect was that of testing $[\mathrm{F}(1,107)=163.46, \mathrm{MSe}=.17]$, with tested items better discriminated $\left(\mathrm{d}^{\prime}=3.35\right)$ than untested items $\left(d^{\prime}=2.64\right)$.

The analyses of hits and false alarms mirrored the $\mathrm{d}^{\prime}$ analysis. Both analyses yielded significant format effects due to pictorial superiority, and also, the hit rate was significantly higher for tested than for untested items. None of these item analyses showed any evidence that subjects in the attribute-memory conditions performed more poorly than subjects in the items-only condition, a finding predicted by the automatic processing model. Light and Berger (1976) also reported this finding and noted their own surprise. However, as in the Light and Berger study, the attribute analysis proves to be very revealing and directly addresses the substantive theoretical concerns of the experiment.

A measure of position memory and a measure of color memory were calculated and analyzed to assess subjects' attribute recall. Position memory was assessed by determining the probability that a subject correctly recalled position conditionalized upon a hit as a function 
Table 2

Mean Final Recognition Performance on d', Hits, False Alarms, Position Attribute, and Color Attribute as a Function of Format (Picture or Word) and Instructions

\begin{tabular}{lccccc}
\hline & \multicolumn{5}{c}{ Dependent Measure } \\
\cline { 2 - 6 } Format & $\mathrm{d}^{\prime}$ & Hits & $\begin{array}{c}\text { False } \\
\text { Alarms }\end{array}$ & $\begin{array}{c}\text { Position } \\
\text { Attribute }\end{array}$ Color \\
Attribute
\end{tabular}

of list and immediate recognition testing. Then, a conditional probability for correct recall of color was similarly calculated for each subject. An analysis of variance was conducted on each measure, with format and instructions as between-groups factors and list and testing as within-subjects factors. The probabilities, as a function of format and instructions, appear in Table 2 .

The analysis of probability of memory for position resulted in a significant format effect due to pictorial superiority $[\mathrm{F}(1,107)=26.67, \mathrm{MSe}=.11]($ means $=.82$ and .71). The effect of instructions was also significant $[\mathrm{F}(3,107)=5.04, \mathrm{MSe}=.11]$. The I-only group had a mean of .69 and thus performed worse than the I-P, I-C, and I-C-P groups, which did not differ from each other with means of $.80, .76$, and .79 . Orthogonal decomposition of the instructions effect supported this conclusion, as the I-only group differed significantly from the other three conditions $[F(1,107)=6.53]$, whereas the I-C and I-P conditions did not differ from the I-C-P group or from each other $\left(F_{s}<1.0\right)$. Thus, subjects in the I-C condition remembered position as well as the I-P subjects, who were actively trying to encode position-a finding that would appear to suggest that position is encoded at no cost once attention is directed to color. Admittedly, this relationship was more marked for pictures than for words, as Table 2 reveals, although the Instructions by Format interaction did not approach significance $[F(3,107)=1.66, p>.17]$, so only the main effect can be interpreted.

In addition, the list main effect was significant $[\mathrm{F}(3,321)=5.45, \mathrm{MSe}=.031]$ due to subjects' enhanced performance across lists. The Instructions by List interaction $[\mathrm{F}(9,321)=3.70, \mathrm{MSe}=.031]$ also reached significance. It occurred because the I-only condition showed a decrease in performance across lists, whereas the reverse was true for the other three conditions. Finally, instructions interacted with testing and list $[F(9,321)=4.11, \mathrm{MSe}=.029]$ due to the particularly marked decrease in performance across lists for I-only subjects on untested items. Since list was rotated across subjects within conditions, confounding is not a likely explanation for these effects. The performance increment across lists in most conditions is a finding incompatible with notions of automaticity. However, the effect most likely is due to subjects' enhanced awareness of task demands across lists, rather than to a genuine learning effect associated with the encoding process.

The attribute analysis of color yielded only a single effect, that of instructions $[F(3,107)=39.16$, MSe $=$ .081]. The I-only and I-P conditions did not differ (means of .53 and .53) and were barely above the level of chance. The I-C and I-C-P conditions performed well above chance and did not differ substantially (means of .77 and .71 , respectively). This finding suggests very clearly that it is effortful to remember color, as subjects remembered it much better when actively trying. Furthermore, the lack of a format effect suggests that it is as difficult to remember the color of a picture as it is to remember the color of a word.

\section{CONCLUSION}

The main findings of the present study were that (1) it is effortful to remember color, (2) spatial recall did not meet the criteria for automatic processing specified by Hasher and Zacks (1979), but memory was well above chance for this dimension, and (3) the spatial location of pictures is better retained than that of comparable words. Each of these findings will be discussed in turn. The color attribute analysis indicated that unless subjects tried to remember color, memory for the attribute was virtually at chance for both pictures and words, indicating that processing was effortful with respect to the intentionality criterion. However, the fact that item recognition was unaffected by encoding instructions would appear to suggest that attending to color required no capacity, as the trading of item information for attribute information should have occurred for an effortful dimension. However, a similar finding of no tradeoff was reported by Light and Berger (1976), who also found otherwise strong evidence for effortful encoding of color. Since item recognition performance was quite good in both their study and the present one, it may be that evidence for trading will occur only on more difficult recognition tasks. This explanation seems likely, as Park, Puglisi, and Lutz (in press) found evidence for subjects' trading item information in favor of attribute information under attribute-intentional conditions when aged subjects were tested. Task demands were apparently significant enough for these subjects to permit a trading effect to emerge. In general, then, the 
present finding confirms the work of Light and Berger on memory for the color of words and extends it to include simple line drawings. Whether or not these effects would generalize to other types of pictorial stimuli is not clear, but the means in the present study for the color of line drawings are so similar to those of Light and Berger for the color of words that generalization would seem to be a likely possibility. Of course, one might find very different effects if color memory were studied as a nonindependent dimension of a stimulus (e.g., a red apple).

The findings with respect to automaticity of position are more complex. As for color, there is no evidence for trading; that is, intentionality to learn position did not decrease item recognition, indicating that position satisfies the capacity criterion. However, position memory was better in the intentional condition than in the incidental condition, so the criterion that intentionality have no effect was not satisfied. The position analysis also indicated that subjects who were intentional for color but incidental for position (I-C group) remembered position just as well as if they intended to learn it. This was particularly true for pictorial stimuli. Although subjects did not automatically encode position if they were merely concentrating on learning items, once their attention was directed toward any attribute (even just color), position was remembered at no additional cost. Park and James (Note 1) reported thr: same finding for children's picture memory, suggesting that the finding is reliable, at least with respect to pictures.

The position memory data can perhaps be best understood if we examine Hasher and Zacks' (1979) contention that stimulus information can be encoded along a continuum of automaticity. The present findings appear to indicate that position is located toward the automatic end of this continuum, but it certainly is not processed completely automatically, particularly when these findings are considered in conjunction with the studies reviewed earlier. However, an acceptance of the continuum conceptualization logically leads one to question what is really meant by a memory process that is "semiautomatic"? How does one specify where along this hypothetical continuum a stimulus dimension belongs?

We should point out that there is other evidence besides the lack of difference for position memory between the I-C and I-P groups that does make the continuum notion appear credible. In all of the encoding conditions in the present study, memory for position was above chance. This finding has been reported by many others, even by those who did not find unambiguous evidence for automaticity per se (e.g., Mandler et al., 1977; Von Wright et al., 1975). The fact that subjects remembered position at nonchance levels without attempting to actively encode it indicates that, although position memory is not automatic, it is information that is particularly easy to remember. Since color was remembered at chance level in color-incidental conditions, it is not likely that the exceptional position memory was due to surreptitious attempts to encode dimensions to which subjects were not required to attend. Also, the repeated immediate testing of only the intentional attributes further decreased the prospect of surreptitious processing. Thus, it does appear that position is encoded with much less effort than color is, but it is not encoded effortlessly-a finding consonant with a continuum approach. Direct support for Hasher and Zack's (1979) concept of a continuum might be garnered if one could devise an experiment utilizing timesharing tasks and determine exactly how much capacity different dimensions require to be processed. The current findings indicate that position would require substantially less capacity than color, and perhaps other dimensions, such as frequency, would require even less.

One other interesting effect reliably occurred in this experiment: The location of pictures was more readily recalled than the location of comparable words, an effect reported earlier by Park (1980). It is reasonable that spatial information would be better retained in pictures, as location of an item in space is more important for semantic processing of a picture than for processing of a matched word. Thus, theories of attribute memory must take into account the characteristics of the stimulus with which the attributes are associated, as well as the qualities of the particular attributes themselves.

Finally, we might note that the fact that format never interacted with instructions suggests that the acquisition process underlying memory for the attributes of pictures and words is the same. Such a finding does not provide obvious support for Paivio's (1971) dual coding theory, which posits different storage mechanisms for pictures and words. At the same time, however, Anderson (1978) and Kosslyn and Pomerantz (1977) have pointed out the difficulty in definitively testing the contrasting models of picture memory (e.g., Paivio's dual coding approach vs. Pylyshyn's, 1973, attribute storage approach), and this finding alone certainly does not resolve this heavily debated issue.

\section{REFERENCE NOTES}

1. Park, D. C., \& James, C. Automaticity and controllability of children's encoding operations for spatial and color attributes. Manuscript submitted for publication, 1981. (Available from D. C. Park, Department of Psychology, University of North Carolina, Charlotte, North Carolina 28223.)

\section{REFERENCES}

Acredolo, L. P., Pick, H. L., \& Olsen, M. G. Environmental differentiation and familiarity as determinants of children's memory for spatial location. Developmental Psychology, 1975, 11, 495-501.

ANDERson, J. R. Arguments concerning representation for mental imagery. Psychological Review, 1978, 85, 249-277. 
Hasher, L., \& Zacks, R. T. Automatic and effortful processes in memory. Journal of Experimental Psychology: General, 1979, 108, 356-388.

Kosslyn, S. M., \& Pomerantz, J. R. Imagery, propositions, and the form of internal representations. Cognitive Psychology, $1977,9,52-76$.

Light, L. L., \& Berger, D. E. Memory for modality: Withinmodality discrimination is not automatic. Journal of Experimental Psychology, 1974, 103, 854-860.

Light, L. L., \& Bergen, D. E. Are there long-term "literal copies" of visually-presented words? Journal of Experimental Psychology: Human Learning and Memory, 1976, 2, 654-662.

Light, L. L., Bengen, D. E., \& Bardales, M. Trade-off between memory for verbal items and their visual attributes. Journal of Experimental Psychology: Human Learning and Memory, 1975, 104, 188-193.

Mandler, J. M., Seegmiller, O., \& Day, J. On the coding of spatial information. Memory \& Cognition, 1977, 5, 10-16.

PAIvio, A. Imagery and verbal processes. New York: Holt, Rinehart \& Winston, 1971.

PARK, D. C. Item and attribute storage of pictures and words in memory. American Journal of Psychology, 1980, 93, 603-615.

Park, D. C., Puglisi, J. T., \& Lutz, R. Spatial memory in older adults: Effects of intention. Journal of Gerontology, in press.

Perlmutter, M., Metzger, R., Nezworski, T., \& Miller, $K$ Spatial and temporal memory in 20 and 60 year olds. Journal of Gerontology, 1981, 36, 59-65.

Pylyshyn, Z. W. What the mind's eye tells the mind's brain: A critique of mental imagery. Psychological Bulletin, 1973, 80, 1-24.

Schulman, A. I. Recognition memory and the recall of spatial location. Memory \& Cognition, 1973, 1, 256-260.

Thonndike, E. L., \& LoRaE, I. The teacher's word book of 30,000 words. New York: Columbia University Press, 1944. Von Wright, J. M., Gebhard, P., \& Karttunen, M. A developmental study of the recall of spatial location. Journal of Experimental Child Psychology, 1975, 20, 181-190.

(Received for publication May 1, 1981; revision accepted July 31,1981 .) 